Original paper

\title{
A new facility for proton radiobiology at the Trento proton therapy centre: Design and implementation
}

\author{
Francesco Tommasino $^{\mathrm{a}, \mathrm{b}, *}$, Marta Rovituso ${ }^{\mathrm{b}}$, Eleonora Bortoli ${ }^{\mathrm{a}, \mathrm{b}}$, Chiara La Tessa ${ }^{\mathrm{a}, \mathrm{b}}$, \\ Giada Petringa ${ }^{\mathrm{c}, \mathrm{d}}$, Stefano Lorentini ${ }^{\mathrm{e}}$, Enrico Verroi ${ }^{\mathrm{b}}$, Yuri Simeonov ${ }^{\mathrm{f}}$, Ulrich Weber ${ }^{\mathrm{g}}$, \\ Pablo Cirrone ${ }^{\mathrm{c}}$, Marco Schwarz ${ }^{\mathrm{b}, \mathrm{e}}$, Marco Durante ${ }^{\mathrm{g}}$, Emanuele Scifoni ${ }^{\mathrm{b}}$ \\ ${ }^{a}$ Department of Physics, University of Trento, Povo, Italy \\ ${ }^{\mathrm{b}}$ Trento Institute for Fundamental Physics and Applications (TIFPA), National Institute for Nuclear Physics, INFN, Povo, Italy \\ ${ }^{\mathrm{c}}$ Laboratori Nazionali del Sud (LNS), National Institute for Nuclear Physics, INFN, Catania, Italy \\ ${ }^{\mathrm{d}}$ Department of Physics and Astronomy, University of Catania, Catania, Italy \\ e Protontherapy Department, Azienda Provinciale per i Servizi Sanitari (APSS), Trento, Italy \\ ${ }^{\mathrm{f}}$ Institute of Medical Physics and Radiation Protection (IMPS), University of Applied Sciences, Giessen, Germany \\ ${ }^{g}$ Biophysics Department, GSI Helmholtzzentrum für Schwerionenforschung, Darmstadt, Germany
}

\section{A R T I C L E I N F O}

\section{Keywords:}

Proton beam

Irradiation facility

Radiobiology

Passive scattering

\begin{abstract}
A B S T R A C T
We present a new facility dedicated to radiobiology research, which has been implemented at the Trento Proton Therapy Centre (Italy).

A dual-ring double scattering system was designed to produce irradiation fields of two sizes (i.e. 6 and $16 \mathrm{~cm}$ diameter) starting from a fix pencil beam at $148 \mathrm{MeV}$. The modulation in depth was obtained with a custommade range modulator, optimized to generate a $2.5 \mathrm{~cm}$ spread-out Bragg peak (SOBP). The resulting irradiation field was characterized in terms of lateral and depth-dose profiles. The beam characteristics and the geometry of the setup were implemented in the Geant4 Monte Carlo (MC) code. After benchmark against experimental data, the MC was used to characterize the distribution of dose-average linear energy transfer (LET) associated to the irradiation field.

The results indicate that dose uniformity above $92.9 \%$ is obtained at the entrance channel as well as in the middle SOBP in the target regions for both irradiation fields. Dose rate in the range from 0.38 to $0.78 \mathrm{~Gy} / \mathrm{min}$ was measured, which can be adjusted by proper selection of cyclotron output current, and eventually increased by about a factor 7. MC simulations were able to reproduce experimental data with good agreement. The characteristics of the facility are in line with the requirements of most radiobiology experiments. Importantly, the facility is also open to external users, after successful evaluation of beam proposals by the Program Advisory Committee.
\end{abstract}

\section{Introduction}

In parallel to the growth in number of proton therapy centres [1], an increasing interest is registered concerning experimental facilities where proton radiobiology studies can be performed. This is confirmed by the recent survey published by the European Particle Therapy network radiobiology group [2]. The motivation lies in the extensive research activity on going in the field. While the physical advantages offered by proton therapy are nowadays established (i.e. improved selectivity in the release of dose due to the Bragg peak profile, which reflects into the possibility to spare healthy tissues compared to photon radiation), several aspects of proton radiobiology need to be better understood $[3,4]$. The interest in the elucidation of biological mechanisms associated with particle irradiation stems from the potential to further improve the effectiveness of radiotherapy treatments $[5,6]$. For instance, the combination of radio- and immunotherapy recently provided impressive and encouraging results, which triggered additional investigation involving also particle irradiation [7]. Many experts in the field support the idea that the next significant improvements in particle therapy effectiveness will arise from exploiting different types of combined treatments [8].

In this framework, the possibility to have a research facility "nextdoor" to a treatment centre sounds as particularly useful. At the Trento proton therapy centre (Agenzia Provinciale per i Servizi Sanitari, APSS)

\footnotetext{
* Corresponding author at: Department of Physics, University of Trento, Via Sommarive, 14 - 38123 Povo (TN), Italy.

E-mail address: francesco.tommasino@unitn.it (F. Tommasino).
} 


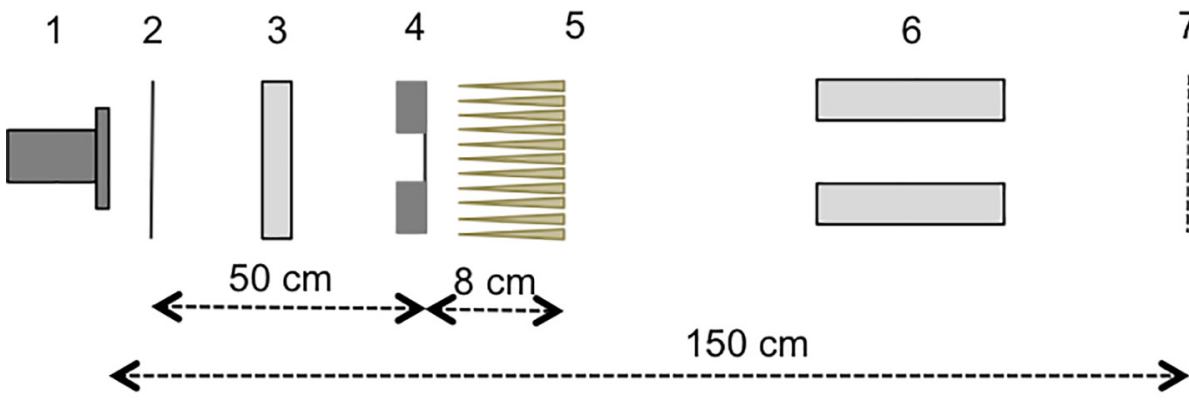

7 Fig. 1. Schematic view of the dual-ring setup (not to scale). The required enlargement of the beam is obtained by the combined effect of the first scattering foil and the double ring. The first foil is positioned just after the end of the beam pipe. The modulator provides energy modulation, in case a SOBP is needed. Plastic collimators can be used in case small irradiation fields are required.

1 Beam pipe

2 First Foil

3 Monitor Chamber
5 Range Modulator

6 Collimation (optional)

7 Target Position

4 Dual Ring

a room dedicated to experimental activities is available, which is equipped with two proton beam lines. Following an institutional agreement with APSS, research activities in the experimental room are managed and supervised by the Trento Institute for Fundamental Physics and Applications (TIFPA), which is part of the Italian National Institute for Nuclear Physics (INFN). Research groups interested in the facility can submit research proposal to the Program Advisory Committee (PAC) organized by TIFPA (http://www.tifpa.infn.it/scinit/med-tech/p-beam-research/). One beam line is dedicated to physics-oriented research, ranging from detector testing to space radioprotection. A fix pencil beam is delivered, which has been recently characterized as summarized in Tommasino et al. [9]. The second beam line is dedicated to radiobiology research. This requires the production of an irradiation field that is large enough to ensure the delivery of dose over an area of several squared centimetres, with dose uniformity above $90 \%$.

For this purpose, an appropriate passive scattering system has been designed and implemented. Starting from the beam parameters measured in air, the lateral modulation is obtained with a dual-ring scattering system, which has been optimized in order to reach the required dose homogeneity over fields of two sizes. Energy modulation is performed with a custom-designed 3D modulator, resulting into an extended spread-out Bragg peak (SOBP) of $2.5 \mathrm{~cm}$. This work presents the results obtained from the experimental characterization of the two irradiation fields. In addition to that, the geometry of the beam line has been fully implemented in the Hadrontherapy advanced example of the open source Geant4 Monte Carlo (MC) code [10]. Results obtained from the benchmark of MC simulations against experimental data are also presented.

\section{Methods}

A proton beam in the energy range between 70 and $228 \mathrm{MeV}$ is available at the Trento proton therapy centre. Beam intensity can be adjusted in the range 1-300 nA (nominal intensity at cyclotron exit). The beam is accelerated to the maximum energy by a cyclotron (Proteus Plus, IBA, Belgium), followed by an energy selection system (ESS) allowing the transport of any energy in the range $70-228 \mathrm{MeV}$ either to the treatment gantries or to the experimental room. Energies below $70 \mathrm{MeV}$ can be obtained by degrading the beam via a pre-absorber positioned at the end of the beam line, just upstream of the target to be irradiated. A dipole magnet at the entrance of the experimental cave allows switching the beam to one of the two available beam lines, where a fix horizontal pencil beam is delivered. The characteristics of the pencil beam in air are summarized in two publications $[9,11]$.

\section{Large field irradiation}

The passive scattering system implemented in the facility is based on the dual-ring design proposed by Takada $[12,13]$. A similar solution was adopted previously by Helmbrecht et al for the Dresden facility $[14,15]$. The system is composed of a first scattering foil (high atomic number) which produces a first enlargement of the beam, followed by a second scattering element characterized by an inner circular region surrounded by a ring structure, the two elements having different scattering power but same water-equivalent thickness (i.e. the dual ring). The different elements (e.g. material composition, thickness and lateral dimension) can be optimized in order to obtain a flat profile at the target position. Compared to a single scattering system, the dualring design is preferable due to the expected increase in beam transport efficiency by about a factor 3 [12].

In order to optimize the system, an initial proton beam energy must be selected. We decided to design our scattering system for a $148 \mathrm{MeV}$ proton beam, which is representative for typical energies used in proton therapy. The distance between the first foil and the dual-ring, as well as that from the beam exit window to the target position, were then fixed at 0.5 and $1.5 \mathrm{~m}$, respectively. The setup implemented in Trento is schematically shown in Fig. 1 (not to scale).

The beam characteristics at $148 \mathrm{MeV}$ nominal energy were previously investigated [11]. In summary, the pencil beam in air has a Gaussian profile at the target position (sigma of about $4.6 \mathrm{~mm}$ ) and a range of $15.24 \mathrm{~cm}$ (distal R80, i.e. depth corresponding to the $80 \%$ of the maximum dose after the peak). The beam line elements were optimized in order to obtain dose uniformity above $90 \%$ in two irradiation fields of about 6 (setup nr. 1) and $16 \mathrm{~cm}$ (setup nr. 2) diameter, respectively. This size is large enough to irradiate common samples used for radiobiology experiments (e.g. tissue flasks, petri dishes). The uniformity of the measured beam profiles was evaluated according to the following formula:

$U[\%]=100 \cdot\left(1-\frac{I_{\max }-I_{\min }}{I_{\max }+I_{\min }}\right)$

where $I_{\min }$ and $I_{\max }$ indicate the minimum and maximum pixel intensity in the region of interest, respectively. Eventually, a plastic collimator can be employed to restrict the irradiation region to a smaller area, reducing at the same time neutron production compared to higher $\mathrm{Z}$ materials [16]. Details on the scattering elements for the two setups are collected in Table 1. 
Table 1

Summary of the characteristics (size and material) of scattering elements for both the setups used in the study.

\begin{tabular}{|c|c|c|c|c|c|c|c|}
\hline \multirow[t]{2}{*}{ Setup Number } & \multicolumn{2}{|l|}{ First Foil } & \multicolumn{3}{|c|}{ Inner Ring } & \multicolumn{2}{|c|}{ Outer Ring } \\
\hline & Material & Thickness (mm) & Material & Thickness (mm) & Diameter (mm) & Material & Thickness (mm) \\
\hline 1 & $\mathrm{~Pb}$ & 0.8 & $\mathrm{~Pb}$ & 1.5 & 11 & $\mathrm{Al}$ & 5 \\
\hline 2 & $\mathrm{~Pb}$ & 3.4 & $\mathrm{~Pb}$ & 5.5 & 22.5 & $\mathrm{Al}$ & 16 \\
\hline
\end{tabular}
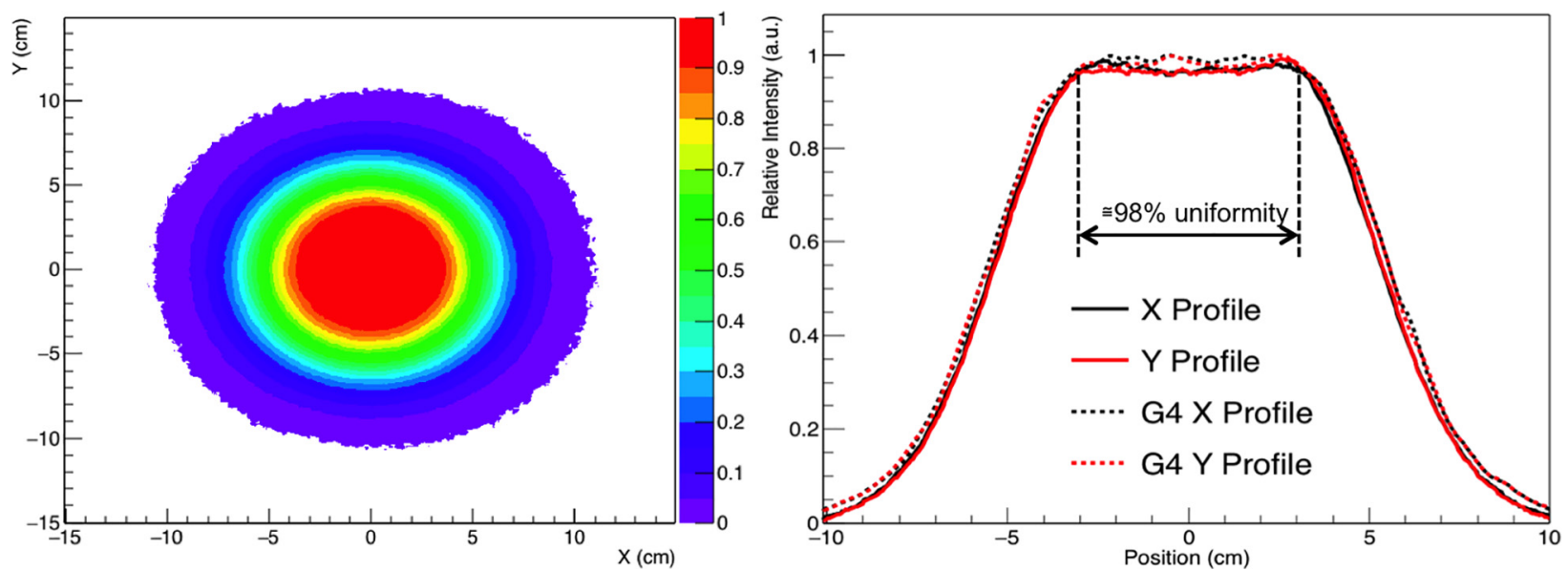

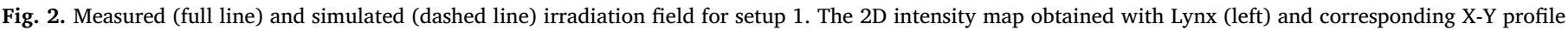
extracted at the centre of the field (right). The $\pm 3 \mathrm{~cm}$ region where about $98 \%$ uniformity was measured is indicated in the figure.

The SOBP was shaped with a 3D-printed range modulator (RM). This modulator is an enhancement of the commonly used ridge filters [17]. It has a much finer and a two-dimensional structure consisting of a matrix of pin-like elements, whose geometry is optimized according to the intended SOBP characteristics. Due to the fine structure of the pinmatrix having a period of $2 \mathrm{~mm}$ it can be used as a static element (no rotation or oscillation needed) that can be positioned close (e.g. $50 \mathrm{~cm}$ ) to the target. The RM used in this work is a special version of the socalled "3D range-modulator" [18], at which all pins have the same shape and height; therefore it is called "2D range modulator".

For this work, starting from a measured Bragg peak curve at $148 \mathrm{MeV}$, the fine geometry contour of the RM pins has been optimized in order to obtain a $2.5 \mathrm{~cm}$ modulated SOBP. The RM was finally manufactured using a high quality 3D-printer (Stratasys Objet30 Pro PolyJet) and an acrylate-based proprietary resin (Duruswhite RGD430). Due to the lower Z compared to metallic RM, the device used in this work provides reduced scattering and beam losses. The passive scattering setup can be used either with or without the RM, according to the needs of the specific experiment.

\section{Detectors}

The characterization of the resulting irradiation field was performed by means of commercial detectors. The lateral profiles at the target position were measured with a scintillating screen coupled to CCDcameras, offering a $0.5 \mathrm{~mm}$ resolution [19] (Lynx, IBA-Dosimetry). Range and profile measurements were carried out with a CC01 ionization chamber (active volume $0.01 \mathrm{~cm}^{3}$ ) inserted in a water phantom (IBA-Dosimetry). EBT3 films (Gafchromic, Ashland Advanced Materials) were employed to determine the surface dose. Films were cut into $3 \times 3 \mathrm{~cm}^{2}$ pieces and positioned at target position corresponding to the centre of the irradiation field. An Epson Perfection V750 Pro flat-bed scanner was employed to digitalize the films. Scans were acquired in transmission mode with a 72 dpi resolution. The analysis was performed only in the red channel of the 48-bit RGB image, due to the highest sensitivity in the dose range of interest [20]. The EBT3 film intensity was converted into dose values by means of a previous calibration. Finally, the Mini-Q detector [21], consisting of a combination of integral and stripped ionization chambers, was used in order to monitor the delivered beam fluxes. As indicated in Fig. 1, this last detector was positioned just after the first scattering foil, in order to collect the whole proton beam. The information on the total delivered beam fluence was used for dosimetry check as described below.

\section{Geant4 simulations}

The experimental setup was reproduced in the Geant4 (version 10.02.p03) advanced example Hadrontherapy class, by implementing the geometry of all elements of the passive scattering line shown in Fig. 1. The source consists of a proton beam originating at the beam exit window with a Gaussian profile of $2.5 \mathrm{~mm}$ sigma and angular divergence of 3.2 and $3.6 \mathrm{mrad}$ in the orthogonal $\mathrm{X}$ and $\mathrm{Y}$ direction, respectively, according to previous measurements [11]. The initial beam energy was set to $147.8 \mathrm{MeV}$, with a Gaussian energy spread equal to $0.8 \mathrm{MeV}$ (standard deviation). This energy is slightly lower compared to the nominal energy of $148 \mathrm{MeV}$, due to minor beam losses taking place during transport of the beam to the experimental room.

Dose and dose-average linear energy transfer (LET) were scored by means of a water phantom detector [22], which can be arbitrarily subdivided into voxels/slices. Specifically, a water phantom of $40 \times 40 \times 40 \mathrm{~cm}^{3}$ was simulated. The phantom was divided into cubic voxels of $1 \mathrm{~mm}$ edge length for the comparison of lateral and depthdose profiles. $5 \times 10^{6}$ primary particles were simulated in each configuration. A higher statistics $\left(10^{8}\right.$ primary particles) was needed to obtain the simulated profiles of Fig. 3. Dose-average LET was scored in the central $\pm 2.5 \mathrm{~cm}$ region, corresponding to the dimension of a typical tissue culture flask, and the voxel thickness in beam direction was increased to $2 \mathrm{~mm}$. This was necessary in order to get statistically stable results. In the following, we will use the term LET to indicate doseaverage LET.

\section{Results}

The results of the characterization campaign will be described here. 

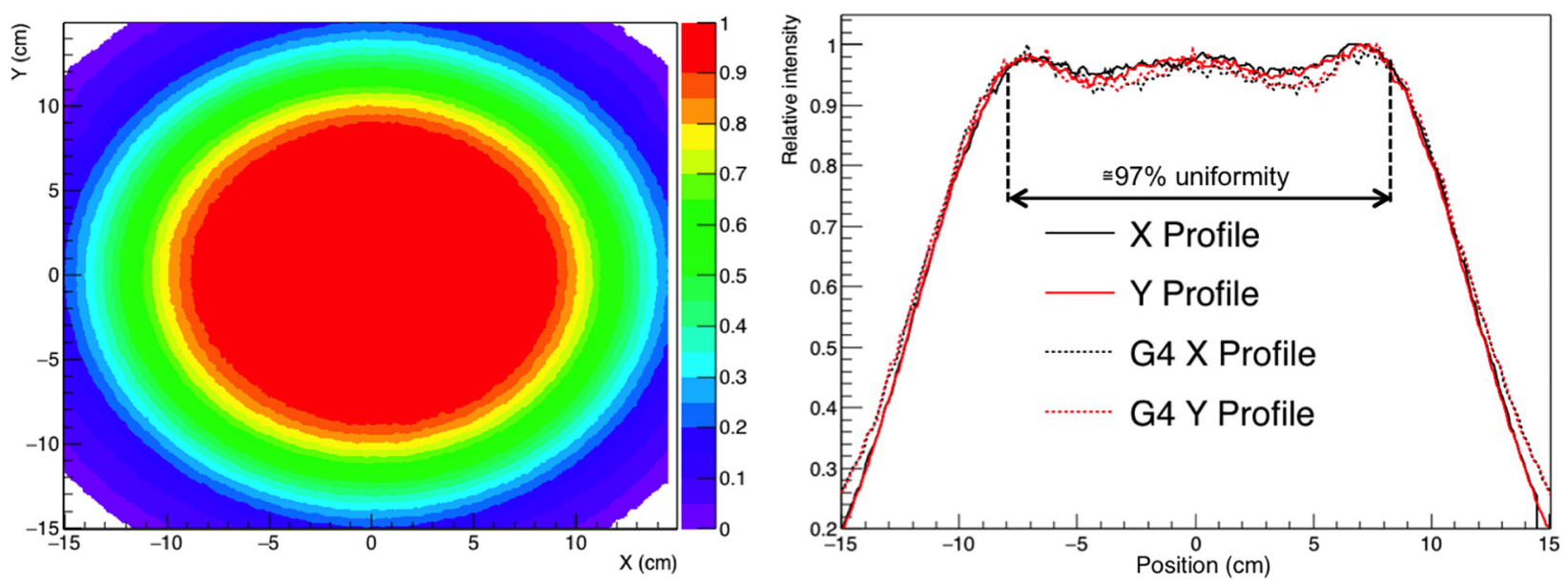

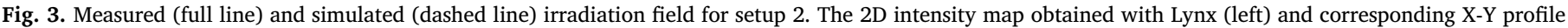
extracted at the centre of the field (right). The $\pm 8 \mathrm{~cm}$ region where about $97 \%$ uniformity was measured is indicated in the figure.

Experimental data will be presented together with simulated ones, providing a benchmark for the MC simulations.

\subsection{Characterization of the irradiation field}

The 2D beam spot measured at the entrance channel for both the setups are shown in Figs. 2 and 3, respectively. X-Y profiles extracted at the spot centre are also presented. When applied to the $\pm 3 \mathrm{~cm}$ central region for setup 1, a uniformity of $98.4 \%$ and $98.1 \%$ was obtained in Xand Y direction, respectively. For the setup 2, the uniformity was evaluated in a larger region $( \pm 8 \mathrm{~cm}$ ) resulting into $97.4 \%$ and $96.5 \%$ in Xand Y direction, respectively. This slightly lower uniformity compared to setup 1 is due to the small "wave effect" observed in the beam profile. This is due to the mixing of the beam components scattered by the inner and outer ring, this effect being more pronounced in larger fields. In any case, uniformity well above the requested $90 \%$ was obtained with both setups.

The simulated profiles are in good agreement with the measured ones, especially in the central part of the field. Slight deviations from experimental data appear in the tails for both setups, which are attributed to sub-optimal simulation of the beam line optics. However, this corresponds to out-of-field irradiation regions, being not relevant for the purpose of this work.

The depth-dose curves acquired with the water phantom setup for both the setups are shown in Fig. 4. The first measured depth in water corresponds to $5.5 \mathrm{~cm}$. This is due to the fact that at the lowest depth the beam had to cross the phantom entrance wall (waterwater equivalent thickness $=1.74 \mathrm{~cm}$ ) and an additional $3 \mathrm{~cm}$ water gap, and also takes into account the effective point of measurement of the CC01 chamber. Pristine Bragg peaks were measured for the setup 1 and 2 without the use of the modulator. The RM was then tested with setup 1 , resulting in a SOBP with the expected modulation of about $2.5 \mathrm{~cm}$. Peak-to plateau ratios equal to about 3.3 and 2.8 were obtained for the pristine Bragg peaks with setup 1 and 2, while a value of about 1.7 was observed with the use of the RM.

The depth-dose curves obtained with Geant4 show a good match with experimental data. Concerning the pristine Bragg peaks in Fig. 4, this indicates that the initial beam energy and energy spread used for the simulation are consistent with the actual ones. The agreement is also good for the SOBP obtained with setup 1, thus confirming also the goodness of the RM geometry implemented in Geant 4.

The water phantom setup was also employed to investigate the irradiation profile at different depths. Specifically, the profiles were measured at two different depths for setup $1(5.5$ and $11.5 \mathrm{~cm})$ and for setup 2 (6 and $8 \mathrm{~cm}$ ), as summarized in Fig. 5. The deepest profiles
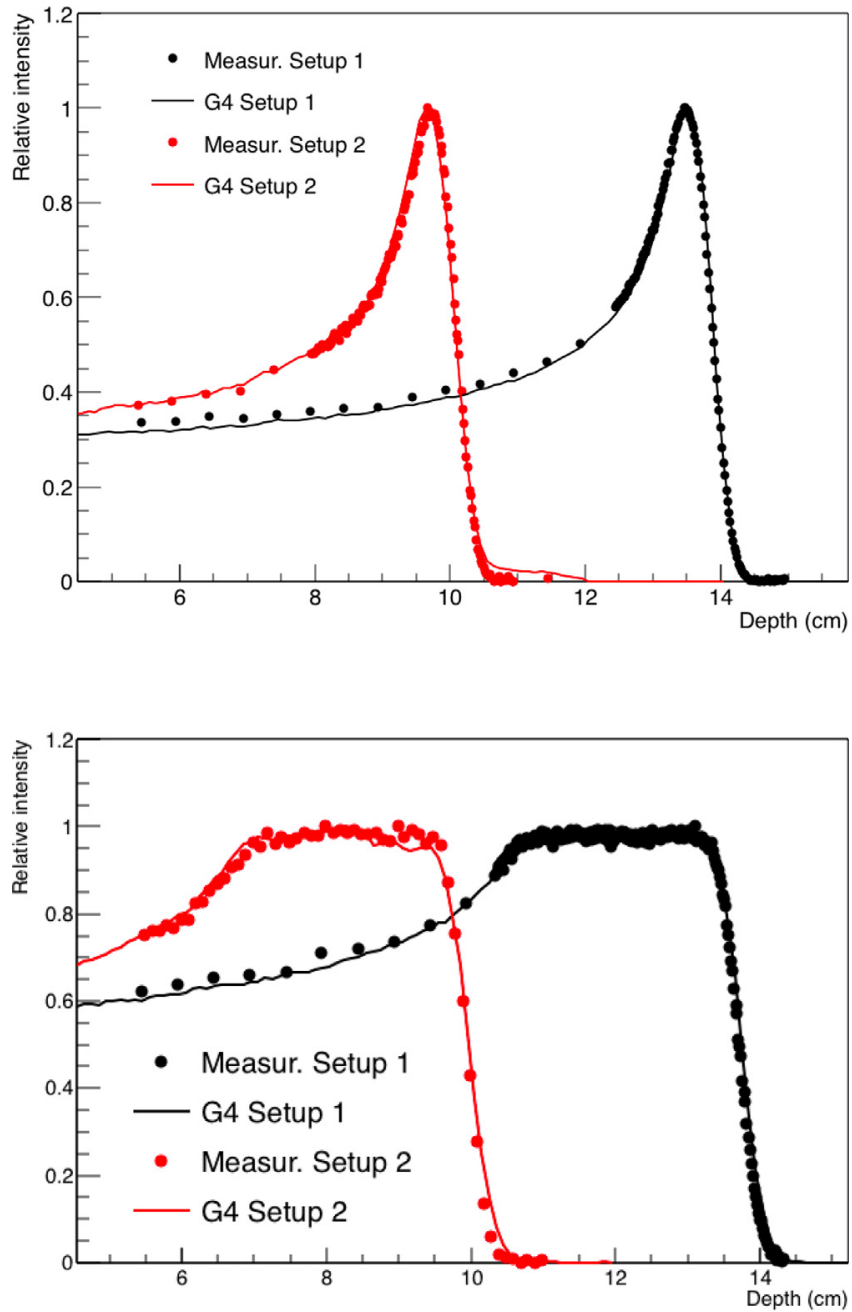

Fig. 4. Experimental depth-dose curves obtained without range modulator (upper panel) and with range modulator (lower panel) for setup 1 and setup 2 .

correspond to mid-SOBP position. The uniformity analysis results into dose uniformity of $96.4 \%$ and $97.7 \%$ in the $\pm 3 \mathrm{~cm}$ for the two positions of setup 1, while uniformity of $92.9 \%$ and $94.9 \%$ are obtained for setup 2 in the $\pm 8 \mathrm{~cm}$ region.

The data shown so far provide also a validation for the geometry and beam parameters implemented in the Geant 4 simulation. Thus, the 

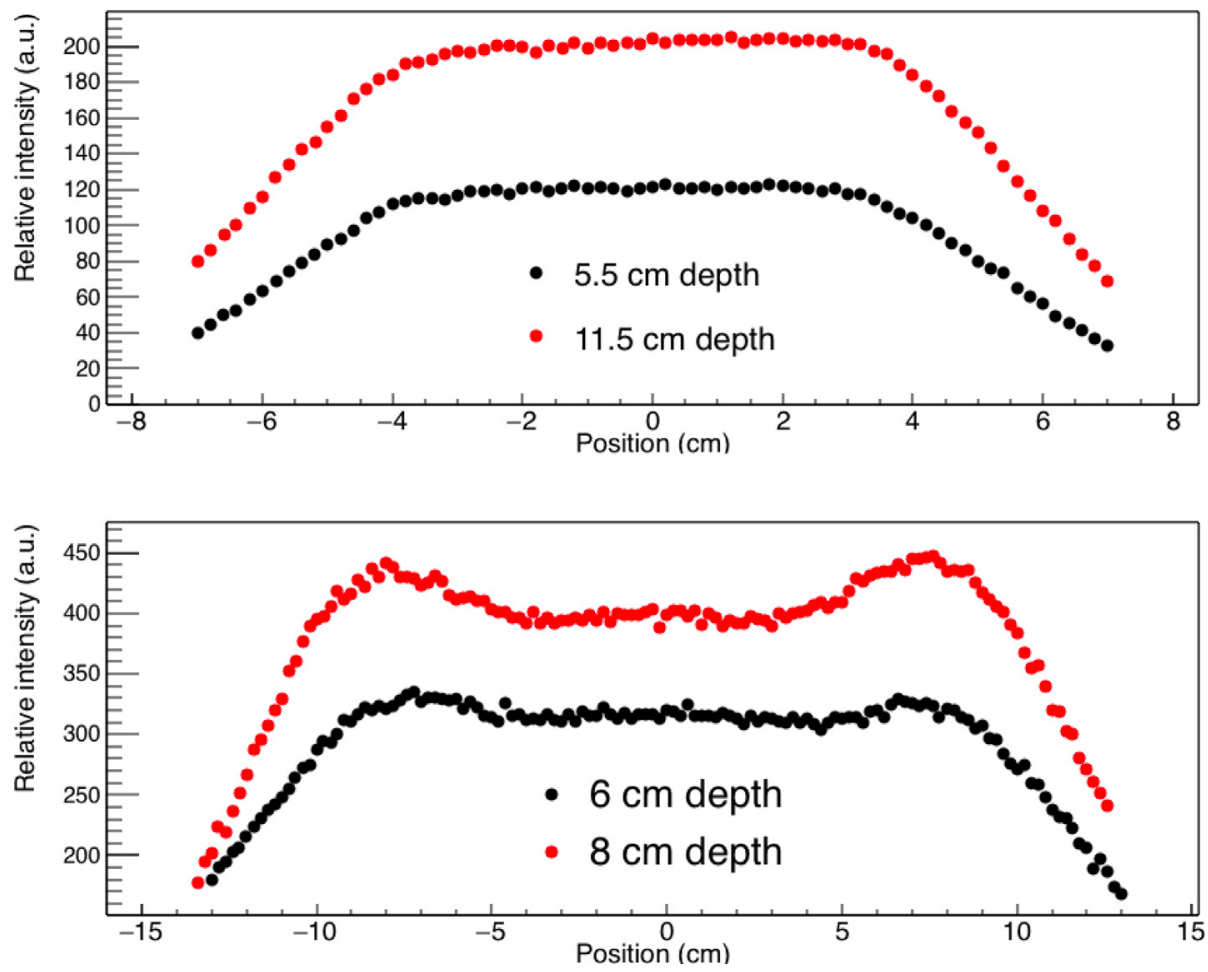

Fig. 5. Profiles of the irradiation field measured with the CCO1 detector placed inside the water phantom for setup 1 (upper panel) and setup 2 (lower panel). A dose uniformity above $94 \%$ was obtained in the $\pm 3 \mathrm{~cm}$ region for setup 1 , while a uniformity above $92 \%$ in the $\pm 8 \mathrm{~cm}$ is observed for setup 2 .
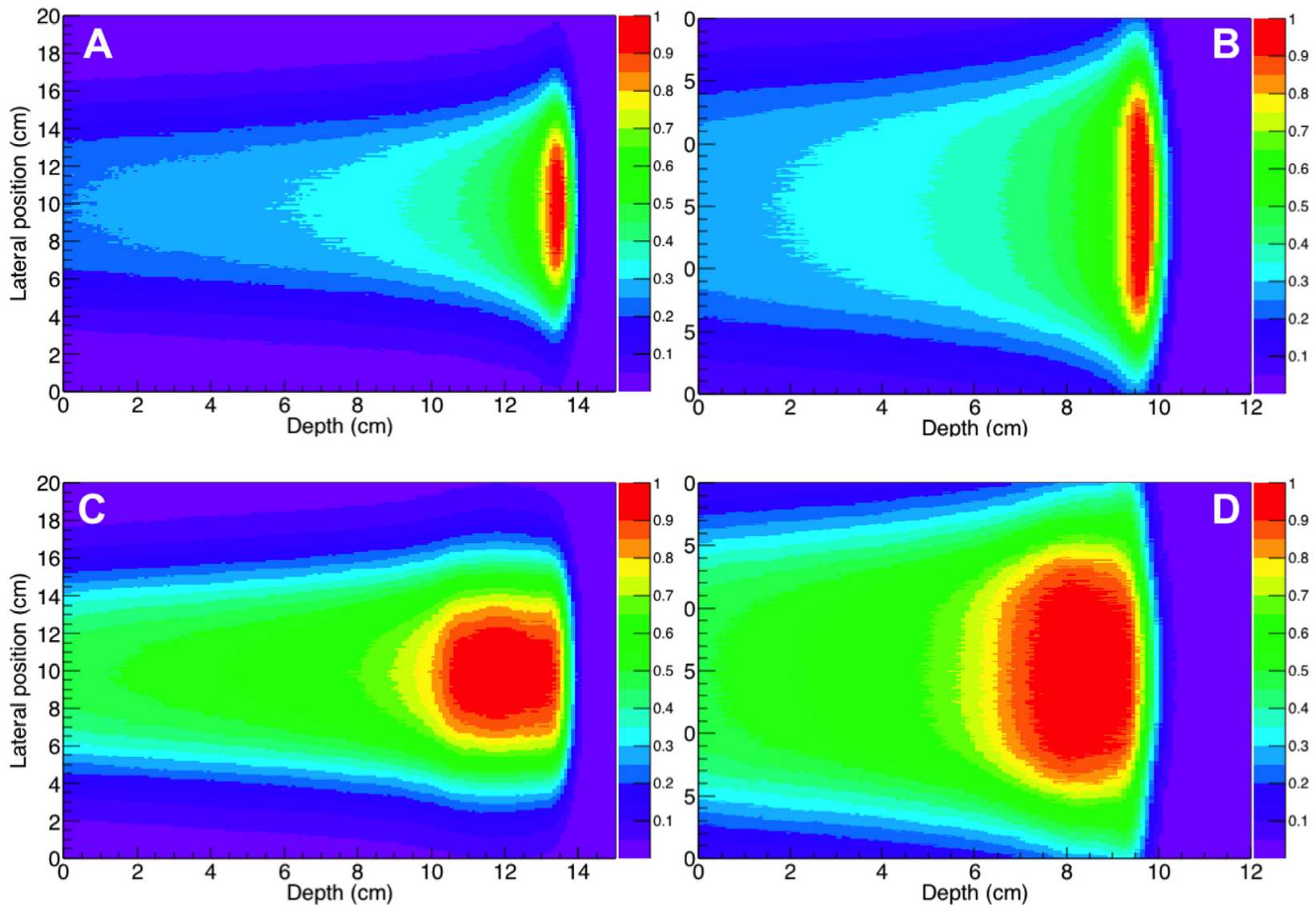

Fig. 6. 2D integrated dose distributions as a function of depth. Data relative to setup 1 without (A) and with (C) the RM are shown on the left, while those relative to setup 2 without (B) and with (D) the RM are shown on the right. Please note the different scale for the $y$-axis for setup 1 and 2. 

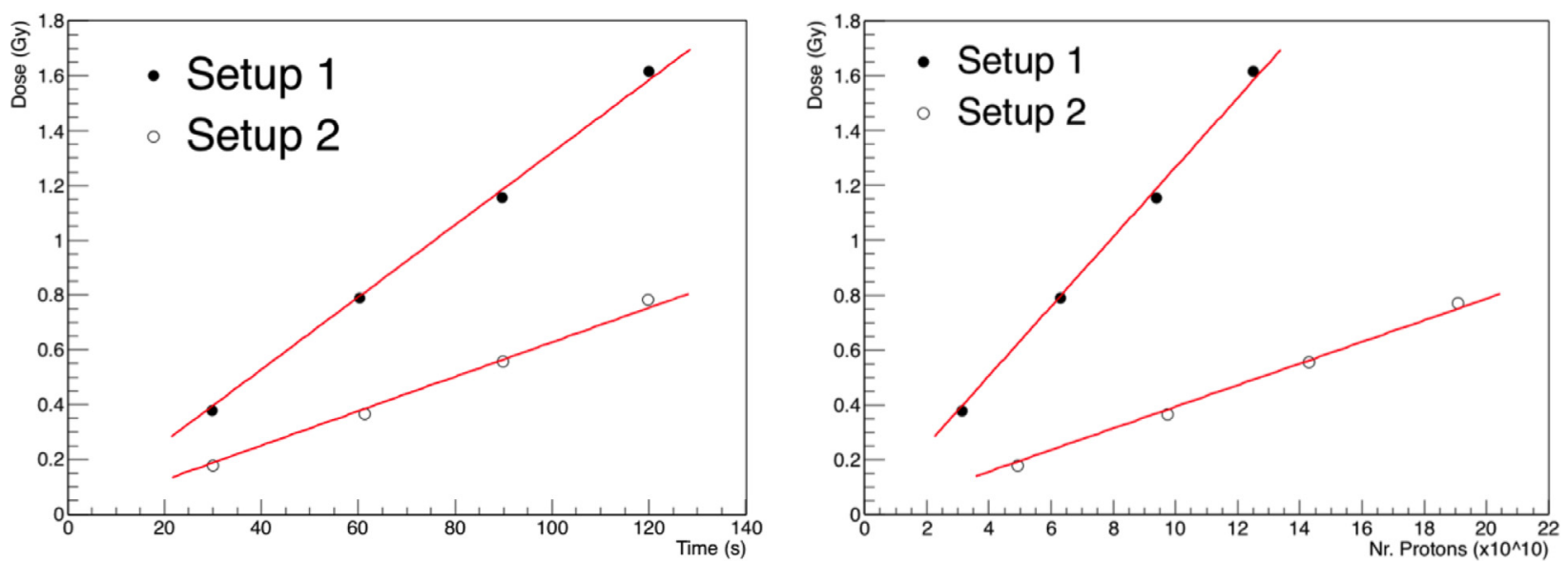

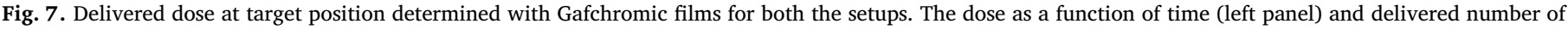
protons (right panel) is shown. The latter was determined with the MiniQ detector positioned just behind the first scattering foil (see also setup in Fig. 1).

MC was used to compute the 2D integrated dose distribution as a function of depth, which is shown in Fig. 6. This was performed for the four possible configurations of the setup, namely for setup 1 and 2, with and without the use of the RM. The plots were obtained by integrating the dose corresponding to single voxels over one lateral direction. Data were then normalized by the maximum dose in the field. This allows the visualization of the complete irradiation field in a single shot, obviously showing the field getting larger for increasing depth and providing information that might be useful for the planning of radiobiology experiments. The dose distributions also show that the distal edge gets curved due to multiple Coulomb scattering in water. This suggests that special care should be adopted when performing experiments at the distal part of the field, eventually including the use of a range compensator to mitigate such effect.

\subsection{Dosimetric characterization}

The study of the dosimetric output obtained with both setups was performed by means of pre-calibrated EBT3 films. The films were positioned at the entrance of the irradiation field (i.e. depth equal to zero). Fig. 7 shows the reconstructed doses obtained for different irradiation times, by requesting a constant beam current. Apart from the good linearity of both data sets, the fit indicates that dose rates of about $0.78 \mathrm{~Gy} / \mathrm{min}(40 \mathrm{nA}$ nominal beam current) and $0.38 \mathrm{~Gy} / \mathrm{min}(80 \mathrm{nA}$ nominal beam current) are obtained with the setup 1 and 2, respectively. These dose rates are compatible with the typical needs of radiobiology experiments and with clinical scenarios. The lower dose rate obtained for the largest field is obviously due to the initial number of protons being distributed on a larger area. Obviously, higher dose rates will be obtained at deeper positions over the SOBP. At the centre of the SOBP, dose rates will increase according to the peak-to-plateau ratios presented above.

The reconstructed doses are displayed in Fig. 7 as a function of the total number of delivered protons as measured by the Monitor Chamber. The fit returns a slope of $1.26 \times 10^{-1} \mathrm{~Gy} / 10^{10}$ protons and $3.93 \times \times 10^{-2} \mathrm{~Gy} / 10^{10}$ protons for the setup 1 and 2 , respectively. These data confirm the linearity in the delivered number of protons as a function of delivery time. At the same time, they also indicate that, provided a preliminary calibration is performed, the proton fluxes measured by the Monitor Chamber can be used to check the dose actually delivered to the sample post-irradiation, a procedure which is presently implemented in the irradiation protocol.

\subsection{LET characterization}

After benchmark against experimental data, Monte Carlo simulations were also used to perform the characterization of dose average LET distribution associated to the irradiation fields. We restricted the analysis to the setup including the RM, being the most relevant configuration for clinical radiobiology studies. LET distributions were investigated at different levels. In the upper row of Fig. 8, we show how LET increases along the depth-dose profile for Setup 1 and 2. LET values in the range $2-4 \mathrm{keV} / \mu \mathrm{m}$ are obtained in the SOBP region, while much higher values are associated with the distal fall-off. The middle row reports the percentage dose distribution as a function of LET over depth for both setups. This is obtained by looking at the simulated target voxel-by-voxel. For each voxel, the depth and the scored LET give the $\mathrm{X}$ and $\mathrm{Y}$ coordinates, respectively, while the dose associated to the voxel is used to fill the $2 \mathrm{D}$ histogram. The histogram is then normalized by the total dose delivered to the target. The colour scale therefore indicates the percentage dose contribution at a given depth and LET. Finally, the lower row of Fig. 8 shows LET spectra at different depths for Setup 1 (left panel) and Setup 2 (right panel). The spectra are obtained by extracting $1 \mathrm{D}$ projections from the $2 \mathrm{D}$ histogram at given depths. Each spectrum was normalized by its integral. This means that each spectrum provides the percentage dose contribution at a given LET. Apart from showing a gradual shift to higher average LET values with depth, the spectra also show that LET distributions get broader with increasing depth, thus reflecting the gradual spread in the energy distribution of the proton beam.

\section{Discussion}

The results presented in this work summarize the efforts dedicated to set-up a new facility for proton radiobiology at the Trento Proton Therapy centre. Based on a relatively simple passive scattering setup, we were able to produce large irradiation fields (i.e. 6 and $16 \mathrm{~cm}$ diameter) that are suitable for carrying out radiobiological studies. This solution has been already proposed in the past for similar purposes $[14,15]$, thus showing the suitability of the dual-scattering setup for radiobiology research, in terms of ease of use, transport efficiency and dosimetric accuracy. The dose homogeneity in the effective irradiation region is above about $93 \%$ for both fields at three different depths. The lowest dose uniformity was obtained for Setup 2 over a $\pm 8 \mathrm{~cm}$ region at $8 \mathrm{~cm}$ depth. This is due to the lateral dual-ring component getting more pronounced, as clearly visible in Fig. 5 (bottom panel). Experiments requiring a large field and higher uniformity should consider a smaller irradiation region (e.g. a dose uniformity of $95.1 \%$ is obtained at $8 \mathrm{~cm}$ depth for Setup 2 in a $\pm 6 \mathrm{~cm}$ region). The dose rate can be finetuned thanks to the extended dynamic range of the cyclotron output current. Specifically, the dose rates obtained with 40-80nA cyclotron output current (i.e. $0.78 \mathrm{~Gy} / \mathrm{min}$ and $0.38 \mathrm{~Gy} / \mathrm{min}$ for the setup 1 and 2, 

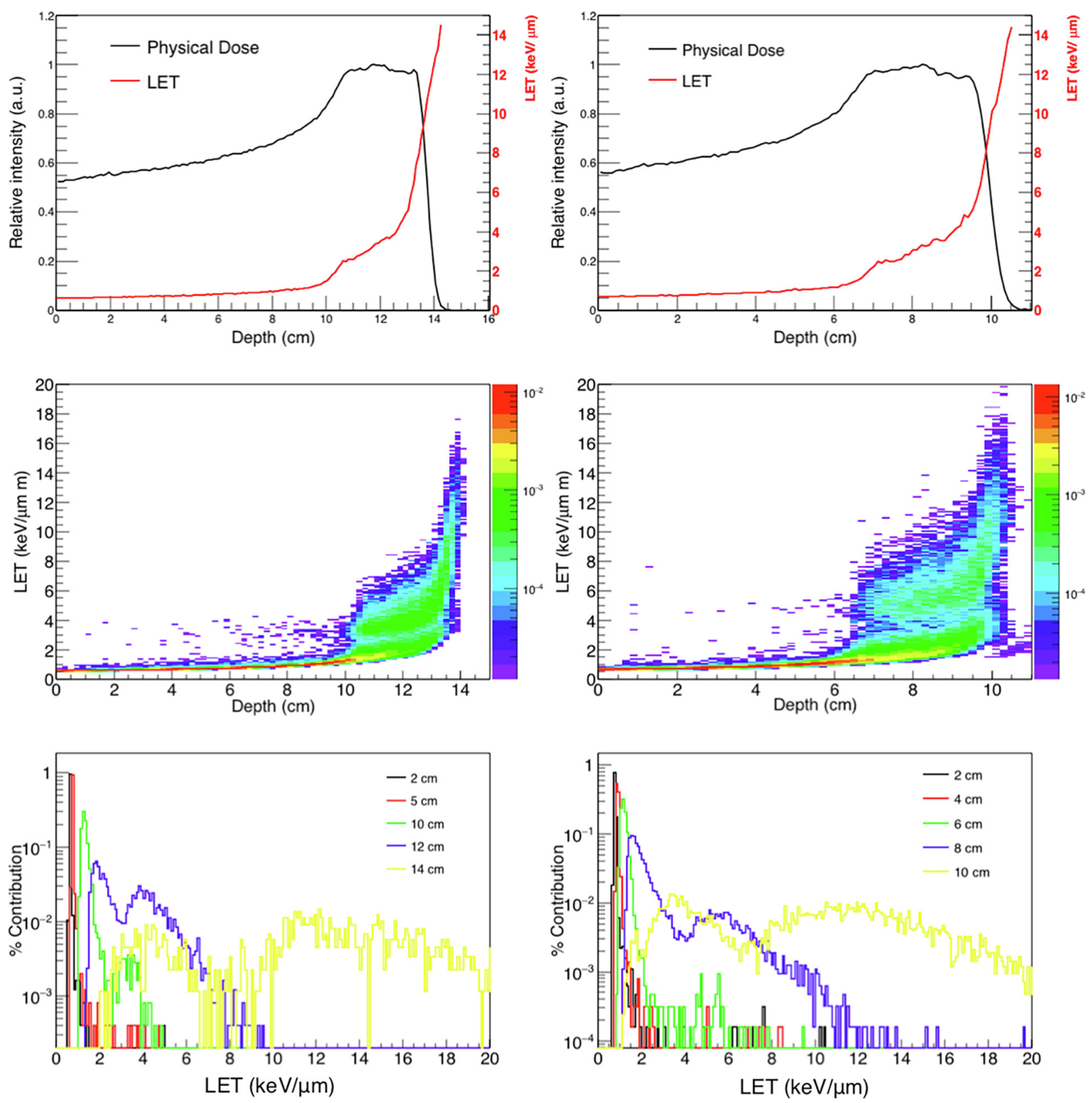

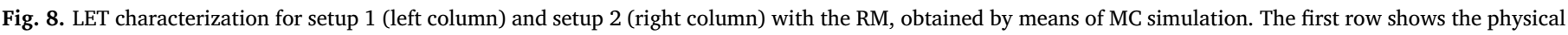

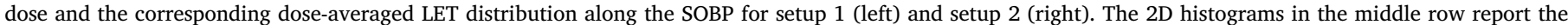

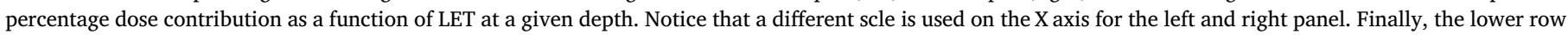
collects LET spectra at selected depths for both setups.

respectively). Taking into account the possibility to require a cyclotron output current in the range 1-300nA, this corresponds to a dose rate at target position ranging from about 0.02 to about $5.8 \mathrm{~Gy} / \mathrm{min}$ and from about 0.01 to about $2.85 \mathrm{~Gy} / \mathrm{min}$ for setup 1 and 2, respectively.

Currently, the setup allows performing in vitro experiments based on the use of either tissue culture flasks or petri dishes, with dimensions in line with the irradiation fields. Samples are aligned at target position thanks to a laser system. Single samples as well as stacks of tissue flasks can be exposed. Stacks of water-equivalent material can be used as preabsorbers, thus selecting different depth positions for irradiation along the SOBP. The facility is equipped with an incubator, which can be used for cell storage before and after the irradiation. A biological laboratory is available on request at the TIFPA institute, which is few kilometres distant from the proton therapy centre. Work is on going in order to extend the use of the facility to the irradiation of small animals.
Adjustable plastic collimators are available and can be used to restrict the irradiation field to smaller target sizes (e.g. a mouse leg, tissue samples or an implanted tumour).

The complete irradiation setup was reproduced in Geant4. Importantly, the beam characteristics and the beam line geometry were implemented in a source file of the Hadrontherapy advanced examples, which will be released with the Geant 4 code thus being available to external users. This will allow planning of future experiments, by taking into account also LET information. Eventually, dose and LET can be combined to estimate proton RBE at a specific irradiation position, for the given cell/tissue under consideration.

Radiobiology of charged particles is a growing field, especially thanks to the advances obtained in recent years in the development of new biological techniques [23,24]. This goes together with the gradual spread of proton therapy centres, which is also accompanied by an 
increase in the number of pathologies treated with protons [1]. Importantly, access to the research beam line is open to external users, after positive evaluation of research proposals by the local PAC.

Finally, the data presented in this work also demonstrate the possibility to setup a proton radiobiology facility in the framework of a clinical facility built by a commercial company and might become a reference for centres interested in radiobiology research.

\section{Acknowledgements}

We thank Dr. Y. Takada for preliminary discussion on the dual-ring setup optimization. This work was partially supported by the INFN CSN5 Call "MoVe IT".

\section{References}

[1] Jermann M. Particle therapy statistics in 2014. Int J Part Ther 2014;1:50-4. https:// doi.org/10.14338/IJPT.14-editorial-2.1.

[2] Dosanjh M, Jones B, Pawelke J, Pruschy M, Sørensen BS. Overview of research and therapy facilities for radiobiological experimental work in particle therapy. Report from the European Particle Therapy Network radiobiology group. Radiother Oncol 2018. https://doi.org/10.1016/j.radonc.2018.03.008.

[3] Girdhani S, Sachs R, Hlatky L. Biological effects of proton radiation: what we know and don't know. Radiat Res 2013;179:257-72. https://doi.org/10.1667/RR2839.1.

[4] Tommasino F, Durante M. Proton radiobiology. Cancers (Basel) 2015;7:353-81. https://doi.org/10.3390/cancers7010353.

[5] Durante M, Orecchia R, Loeffler JS. Charged-particle therapy in cancer: clinical uses and future perspectives. Nat Rev Clin Oncol 2017;14:483-95. https://doi.org/10. 1038/nrclinonc.2017.30.

[6] Durante M, Debus J. Heavy charged particles: does improved precision and higher biological effectiveness translate to better outcome in patients? Semin Radiat Oncol 2018;28:160-7. https://doi.org/10.1016/j.semradonc.2017.11.004.

[7] Durante M, Brenner DJ, Formenti SC. Does heavy ion therapy work through the immune system? Int J Radiat Oncol Biol Phys 2016;96:934-6. https://doi.org/10 1016/j.ijrobp.2016.08.037.

[8] Lühr A, von Neubeck C, Pawelke J, Seidlitz A, Peitzsch C, Bentzen SM, et al. "Radiobiology of Proton Therapy": Results of an international expert workshop. Radiother Oncol 2018;128:56-67. https://doi.org/10.1016/j.radonc.2018.05.018.

[9] Tommasino F, Rovituso M, Fabiano S, Piffer S, Manea C, Lorentini S, et al. Proton beam characterization in the experimental room of the Trento Proton Therapy facility. Nucl Instruments Methods Phys Res Sect A Accel Spectrometers, Detect Assoc Equip 2017;869:15-20. https://doi.org/10.1016/j.nima.2017.06.017.

[10] Agostinelli S, Allison J, Amako K, Apostolakis J, Araujo H, Arce P, et al. Geant4-a simulation toolkit. Nucl Instruments Methods Phys Res Sect A Accel Spectrometers, Detect Assoc Equip 2003;506:250-303. https://doi.org/10.1016/S0168-9002(03)
01368-8.

[11] Tommasino F, Rovituso M, Lorentini S, La Tessa C, Petringa G, Cirrone P, et al. Study for a passive scattering line dedicated to radiobiology experiments at the trento proton therapy center. Radiat Prot Dosimet 2018. https://doi.org/10.1093/ rpd/ncy238.

[12] Takada Y. Dual-ring double scattering method for proton beam spreading. Jpn J Appl Phys 1994;33:353-9. https://doi.org/10.1143/JJAP.33.353.

[13] Takada Y. Optimum solution of dual-ring double-scattering system for an incident beam with given phase space for proton beam spreading. Nucl Instruments Methods Phys Res Sect A Accel Spectrometers, Detect Assoc Equip 2002;485:255-76. https://doi.org/10.1016/S0168-9002(01)02137-4.

[14] Helmbrecht S, Baumann M, Enghardt W, Fiedler F, Krause M, Lühr A. Lühr Design and implementation of a robust and cost-effective double-scattering system at a horizontal proton beamline. T11001-T11001 J Instrum 2016;11. https://doi.org/ 10.1088/1748-0221/11/11/T11001.

[15] Beyreuther E, Baumann M, Enghardt W, Helmbrecht S, Karsch L, Krause M, et al. research facility for radiobiological studies at the university proton therapy dresden. Int J Part Ther 2018;5:172-82. https://doi.org/10.14338/IJPT-1800008.1.

[16] Brenner DJ, Elliston CD, Hall EJ, Paganetti H. Reduction of the secondary neutron dose in passively scattered proton radiotherapy, using an optimized pre-collimator/ collimator. Phys Med Biol 2009;54:6065-78. https://doi.org/10.1088/0031-9155/ $54 / 20 / 003$.

[17] Gardey KU, Oelfke U, Lam GK. Range modulation in proton therapy-an optimization technique for clinical and experimental applications. Phys Med Biol 1999;44:N81-8.

[18] Simeonov Y, Weber U, Penchev P, Ringbæk TP, Schuy C, Brons S, et al. 3D rangemodulator for scanned particle therapy: development, Monte Carlo simulations and experimental evaluation. Phys Med Biol 2017;62:7075-96. https://doi.org/10. 1088/1361-6560/aa81f4.

[19] Russo S, Mirandola A, Molinelli S, Mastella E, Vai A, Magro G, et al. Characterization of a commercial scintillation detector for 2-D dosimetry in scanned proton and carbon ion beams. Phys Medica 2017;34:48-54. https://doi.org/10. 1016/j.ejmp.2017.01.011.

[20] Devic S, Seuntjens J, Sham E, Podgorsak EB, Schmidtlein CR, Kirov AS, et al. Precise radiochromic film dosimetry using a flat-bed document scanner. Med Phys 2005;32:2245-53. https://doi.org/10.1118/1.1929253.

[21] Braccini S, Cirio R, Donetti M, Marchetto F, Pittà G, Lavagno M, et al. Segmented ionization chambers for beam monitoring in hadrontherapy. Mod Phys Lett A 2015;30:1540026. https://doi.org/10.1142/S021773231540026X.

[22] Romano F, Cirrone GaP, Cuttone G, Rosa F, Di Mazzaglia SE, Petrovic I, et al. Carlo study for the calculation of the average linear energy transfer (LET) distributions for a clinical proton beam line and a radiobiological carbon ion beam line. Phys Med Biol 2014;59:2863-82. https://doi.org/10.1088/0031-9155/59/12/2863.

[23] Durante M. New challenges in high-energy particle radiobiology. Br J Radiol 2014;87:20130626. https://doi.org/10.1259/bjr.20130626.

[24] Story MD, Durante M. Radiogenomics. Med Phys 2018;45:e1111-22. https://doi. org/10.1002/mp.13064. 